\title{
BANQUES DE TURQUIE : ENTRE DYNAMISME ET TENSIONS
}

\section{Eray Yücel}

Association d'économie financière | «Revue d'économie financière »

2019/4 N 136 | pages 139 à 157

ISSN 0987-3368

ISBN 9782376470366

Article disponible en ligne à l'adresse :

https://www.cairn.info/revue-d-economie-financiere-2019-4-page-139.htm

Distribution électronique Cairn.info pour Association d'économie financière.

(C) Association d'économie financière. Tous droits réservés pour tous pays.

La reproduction ou représentation de cet article, notamment par photocopie, n'est autorisée que dans les limites des conditions générales d'utilisation du site ou, le cas échéant, des conditions générales de la licence souscrite par votre établissement. Toute autre reproduction ou représentation, en tout ou partie, sous quelque forme et de quelque manière que ce soit, est interdite sauf accord préalable et écrit de l'éditeur, en dehors des cas prévus par la législation en vigueur en France. Il est précisé que son stockage dans une base de données est également interdit. 


\title{
BANQUES DE TURQUIE : ENTRE DYNAMISME ET TENSIONS
}

\author{
ERAY YÜCEL*
}

7 homas Jefferson, troisième président américain, a déclaré un jour : "Je crois sincèrement que les institutions bancaires sont plus dangereuses que des armées prêtes au combat. » Cette allégation, aussi figurative soit-elle, se garde toutefois bien de nier le fait que les établissements bancaires sont indispensables aux sociétés modernes. Le début de la modernisation républicaine, qui a suivi la fin de la modernisation ottomane, illustre bien cela. Depuis cette époque, de nombreuses banques ont fleuri en Turquie et contribué à la bonne affectation des capitaux pendant plus d'un siècle. La Turquie d'aujourd'hui, entité majeure du bassin méditerranéen, est réputée posséder un secteur bancaire bien développé. Pour autant, le chemin qui a conduit aux perspectives actuelles n'a pas été un processus exempt de difficultés, pas plus que le système bancaire turc n'est exempt de problèmes. Au contraire, une évaluation minutieuse est nécessaire pour bien comprendre les enjeux qui le touchent, et c'est là l'unique objet de cet article ${ }^{1}$.

En raison de la nature même du sujet, nous optons pour une approche relativement éclectique. Nous commençons ainsi par examiner l'évolution du cadre juridique et technique relatif aux activités bancaires et soulignons les principales étapes s'y rapportant. Ensuite, nous nous penchons sur le passé du système bancaire turc en recourant à divers agrégats et indicateurs économiques choisis. Enfin, nous nous intéressons aux perspectives actuelles des banques exerçant des activités en Turquie et analysons les risques auxquels elles sont confrontées par rapport aux perspectives qui leur sont offertes.

* Département d'économie, Université Bilkent-Ihsan Doğramaci, Ankara. Contact : eray@bilkent.edu.tr. 


\section{ÉTABLIR LE CADRE JURIDIQUE}

La loi bancaire $\mathrm{n}^{\circ}$ 5411, adoptée le 19 octobre 2005 et mise en œuvre le $1^{\text {er }}$ novembre 2005 (Journal officiel, 2005), a été perçue comme un développement salutaire par une majorité d'économistes turcs. La précédente loi avait souvent été critiquée en raison de l'insuffisance de son champ d'application, de la mosaïque de règles qui la composait et de la nécessité constante de la compléter par quantité d'arrêtés et de jugements discrétionnaires. Sans surprise, une telle structure a donné lieu à la possibilité d'un grand nombre d'interventions politiques dont ont profité les gouvernements de l'époque ${ }^{2}$. La loi $\mathrm{n}^{\mathrm{O}} 5411$ a quant à elle fourni le premier plan détaillé depuis plusieurs décennies. Ainsi les capacités des banques et les règles de conduite qui régissent leurs interactions avec le Fonds d'assurance des dépôts d'épargne (TMSF) et l'Agence de réglementation et de surveillance bancaires (BDDK) ont été clairement définies. La nouvelle loi relative à la banque centrale ( ${ }^{\circ}$ 4651, adoptée le 25 avril 2001 et mise en ouvre le 5 mai 2001 ; Journal officiel, 2001), postérieure à la crise turque de 2001, doit également être mentionnée en raison du rôle qu'elle a joué dans la production d'un cadre institutionnel cohérent. Davantage surveillé par l'Autorité turque de la concurrence pour ce qui est des fusions, des acquisitions et des pratiques de fixation des prix, le secteur est aujourd'hui doté d'un cadre juridique capable de fonctionner de manière efficace et autonome. On peut considérer cette évolution comme un pas-de-géant par rapport à l'époque où la Banque centrale (dont l'indépendance date seulement de 2001) et le Trésor (un organisme gouvernemental par définition) exerçaient conjointement et de manière incohérente les pouvoirs de contrôle et de surveillance.

Le système financier turc a toujours été dominé par les banques (autrement dit, les crédits bancaires représentent le principal mode de financement) dans le contexte d'un cadre juridique essentiellement continental. Pour cette raison, le système affiche un scepticisme intrinsèque à l'égard de la liberté de l'innovation financière et lui oppose un certain nombre de barrières. Cette configuration, contrairement à une structure juridique anglo-américaine, nécessite que toute pratique financière soit parfaitement définie par les régulateurs. Cela limite les possibilités d'activités des banquiers turcs, tout en permettant de tirer une leçon des crises bancaires du passé.

Par ailleurs, les liens forts qui unissent le pays au reste du monde, en particulier à l'Europe, aident les régulateurs à adopter presque sans délai toutes les réglementations ultramodernes approuvées à l'échelle internationale. Les banques de Turquie sont ainsi soumises aux 
Accords de Bâle, appliqués dans un grand nombre de pays. La mise en œuvre des normes de Bâle II a connu une franche réussite dans le pays et l'harmonisation avec les normes de Bâle III est en cours. De la même façon, le cadre actuel permet à des banques étrangères d'ouvrir leurs propres succursales ou de détenir des parts dans des banques turques à divers degrés. De jure, comme l'ont montré en détail Togan et al. (2010), le secteur bancaire turc est désormais correctement régulé en ce qui concerne (1) les restrictions sur les banques et sur les liens avec le commerce, (2) les restrictions d'entrées et les règles de sortie, (3) les exigences de réserves, (4) les exigences de capital, (5) les pouvoirs de surveillance, (6) les filets de sécurité, (7) le prêteur en dernier ressort, (8) la surveillance du marché et (9) la propriété gouvernementale ${ }^{3}$.

Une fois les caractéristiques juridiques et réglementaires mentionnées ci-dessus assurées, le principal développement de la dernière décennie a été l'expansion de la «banque participative ». Conformes au principe islamique de l'«interdiction de l'intérêt ", les banques participatives font partie intégrante du système bancaire turc depuis des décennies, pour un volume d'affaires presque confidentiel. De tendance islamique, les gouvernements successifs du Parti de la justice et du développement (depuis novembre 2002) ont ensuite fourni le cadre juridique permettant aux banques participatives de collecter des dépôts en or, ce dernier étant l'actif privilégié de nombreux foyers de droite religieusement conservateurs. Cette étape, abstraction faite de l'aspect négatif des ententes politiques entre le parti au pouvoir et les banques, a été primordiale pour attirer un important volume d'avoirs en or dans le système financier, avoirs qui seraient autrement conservés dans des bas de laine (cf. graphiques 2 à 5 infra).

Au final, il ne serait pas inexact d'affirmer que l'internationalisation et la régulation nationale se sont mutuellement renforcées dans le cas de la Turquie (Togan et al., 2010). Ainsi "la hausse des entrées étrangères en provenance de pays n'ayant pas de lacunes en matière de réglementation et de surveillance soutient le cadre du secteur financier " et "renforce la crédibilité des règles couvrant les barrières au commerce et à l'établissement, l'adéquation des fonds propres, l'assurance dépôts, les comptes consolidés et la surveillance, la libéralisation des mouvements de capitaux et la dérégulation des taux d'intérêt ». Dans le même esprit, l'existence d'une économie de marché fonctionnelle, associée aux critères économiques de Copenhague, requiert - outre une disponibilité et une abordabilité des services financiers, des financements par le biais des marchés boursiers locaux, une facilité d'accès aux prêts et une disponibilité du capital-risque - une certaine «solidité des banques" (Guler, 2019). 
LE SECTEUR BANCAIRE TURC, RÉTROSPECTIVEMENT ${ }^{4}$

D'après la BDDK (2019a), il existe 34 banques de dépôts, 13 banques de développement et d'investissement et 6 banques participatives en Turquie. Ces banques publiques, privées et étrangères opèrent avec 3923,3948 et 3669 succursales, et comptent 66441 , 72212 et 67704 employés, respectivement. Les actifs totaux du secteur s'élèvent à 4139 milliards de livres turques et représentent environ $105 \%$ du PIB. En juin 2019, les banques publiques, les banques privées nationales et les banques étrangères détenaient 39,9\%, 24,2 \% et $35,9 \%$ des actifs bancaires, respectivement. Le secteur évolue actuellement dans un environnement macroéconomique de faible croissance depuis août 2018 (même si la Turquie a affiché l'un des taux de croissance les plus élevés des pays de l'OCDE et du G20), d'inflation instable sans pour autant être chroniquement élevée et de dépréciation monétaire ponctuellement forte, où la politique monétaire a récemment fait l'objet de vives critiques (cf. graphiques 1 infra).

\section{Graphiques 1 \\ Panorama macroéconomique, janvier 2002 à juin 2019}

Graphique 1a

Taux de change livre turque-dollar

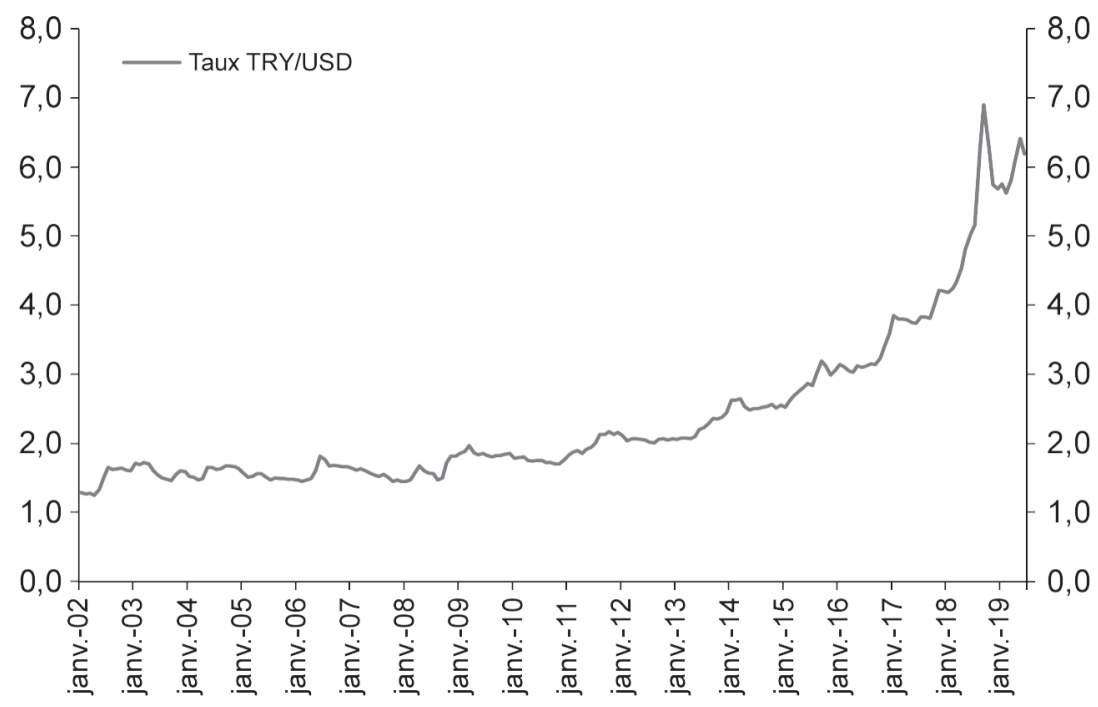




\section{Graphique 1b \\ Taux directeur de la banque centrale}

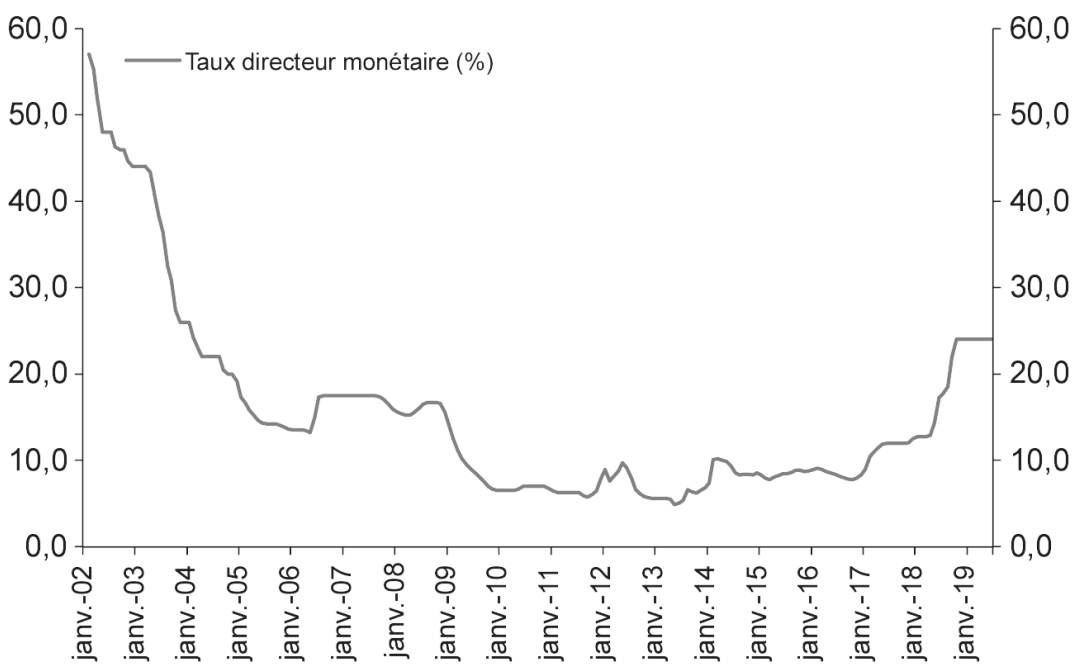

Note : dans le graphique $1 \mathrm{~b}$, le taux directeur a été compilé en appliquant l'approche d'Avci et Yucel (2017).

Source des deux graphiques : données ont été collectées via le système électronique de communication de données de la Banque centrale de la République de Turquie.

Les actifs ont connu une croissance stable dans le secteur, avec des signes avant-coureurs de substitution monétaire. Du côté des banques de dépôts, tandis que la valeur en livres turques des actifs des banques privées nationales diminue, celle des banques étrangères a enregistré une hausse après 2014 (cf. graphiques 2 infra). Dans l'ensemble, les prêts affichent un tableau similaire à celui des actifs. Le ratio "TRY/devises des prêts " a récemment diminué - témoignant d'une intensification de la dollarisation - à la suite d'une hausse antérieure jusqu'à un plateau pour ce qui est des prêts à court terme des banques de dépôts détenues par l'État, des banques étrangères, des banques de dépôts privées nationales, des banques de dépôts étrangères et du secteur bancaire dans sa globalité . Du côté des banques participatives, la répartition des devises est juste en train de se normaliser à la suite des valeurs très élevées qu'elle a atteintes au début des années 2000. Qui plus est, les ratios de prêts improductifs, après avoir été corrigés dans l'environnement de stabilisation post-2001, ont connu une nouvelle détérioration après 2017 (cf. graphiques 3 infra). Un coup d'œil aux dépôts montre une situation comparable à celle des actifs et des prêts et le financement externe des banques par le biais de syndications dans le temps suggère une hausse considérable pendant la période postLehman (cf. graphique 4 infra). 


\section{Graphiques 2 \\ Actifs bancaires, décembre 2002-juin 2019}

Graphique 2a

Volume des actifs avec décomposition en devises

[en milliards]

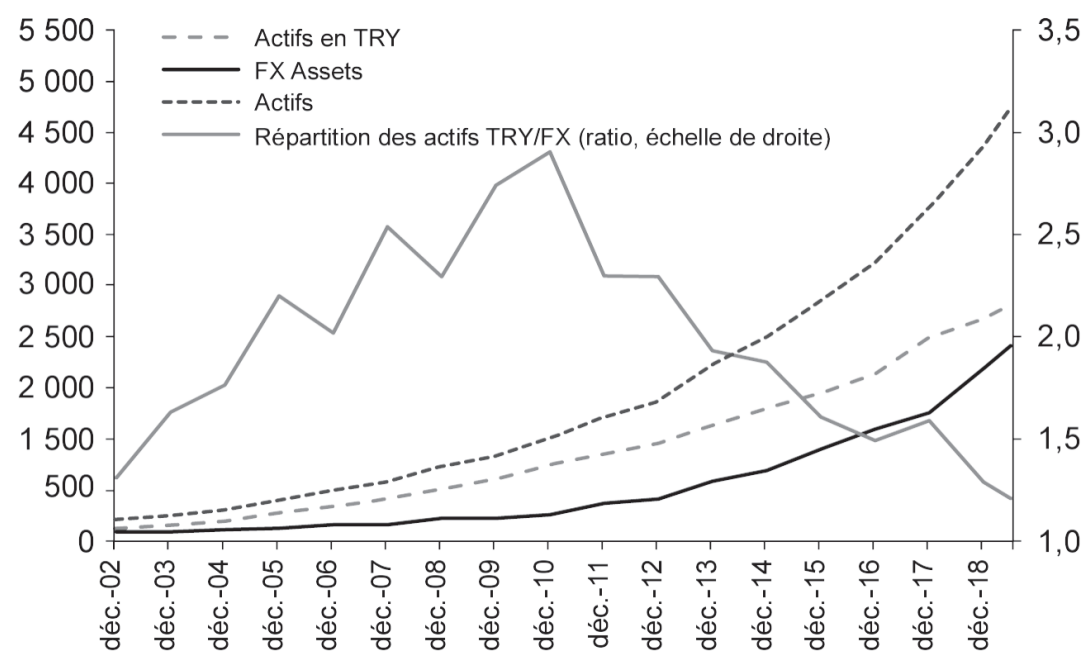

\section{Graphique 2b}

Ventilation des actifs entre banques de dépôts et banques participatives [en milliards]

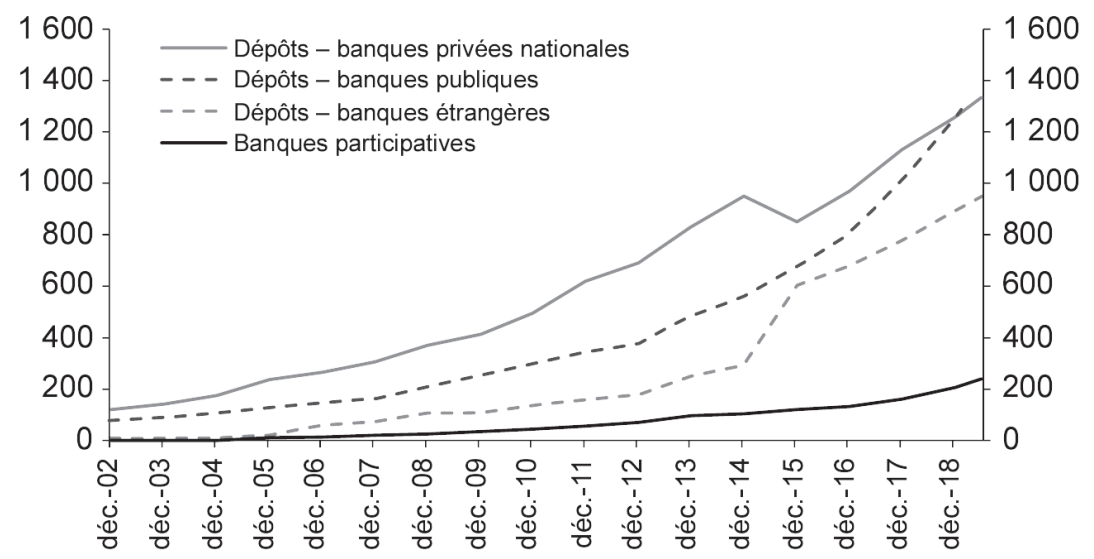

Note : sauf indication contraire, les agrégats s'entendent en livres turques.

Source des deux graphiques : données collectées auprès de l'Agence de réglementation et de surveillance bancaire. 


\section{Graphiques 3 \\ Prêts, décembre 2002-juin 2019}

Graphique 3a

Volume des prêts avec décomposition en devises

[en milliards]

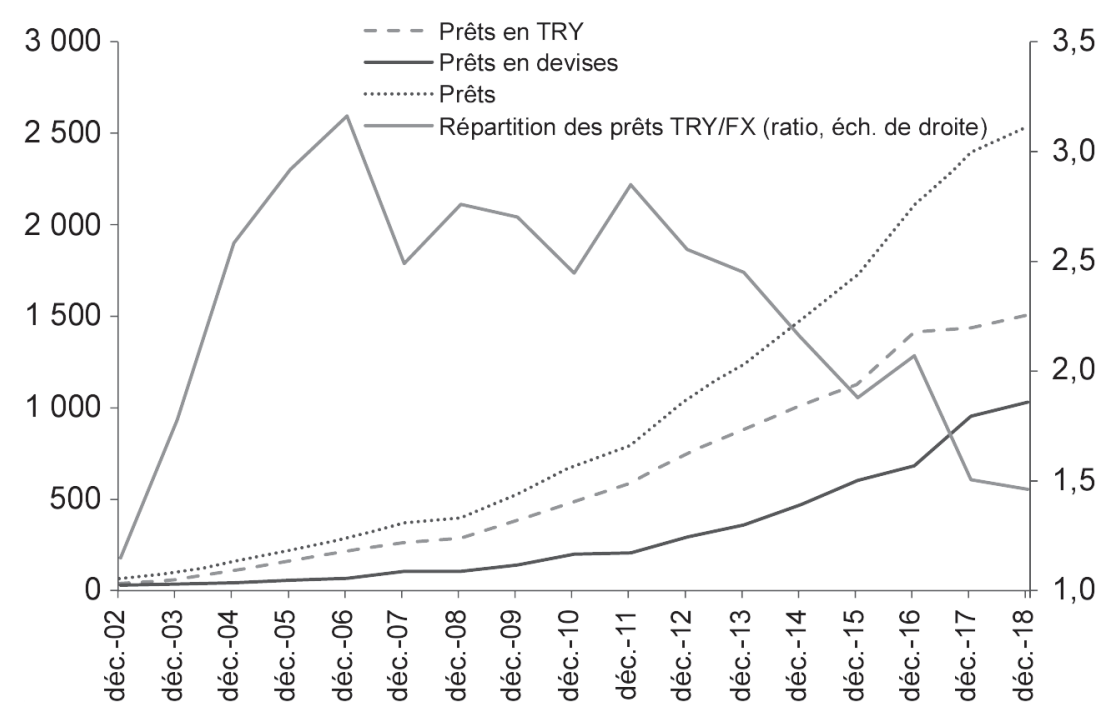

\section{Graphique 3b}

\section{Ratios des prêts improductifs}

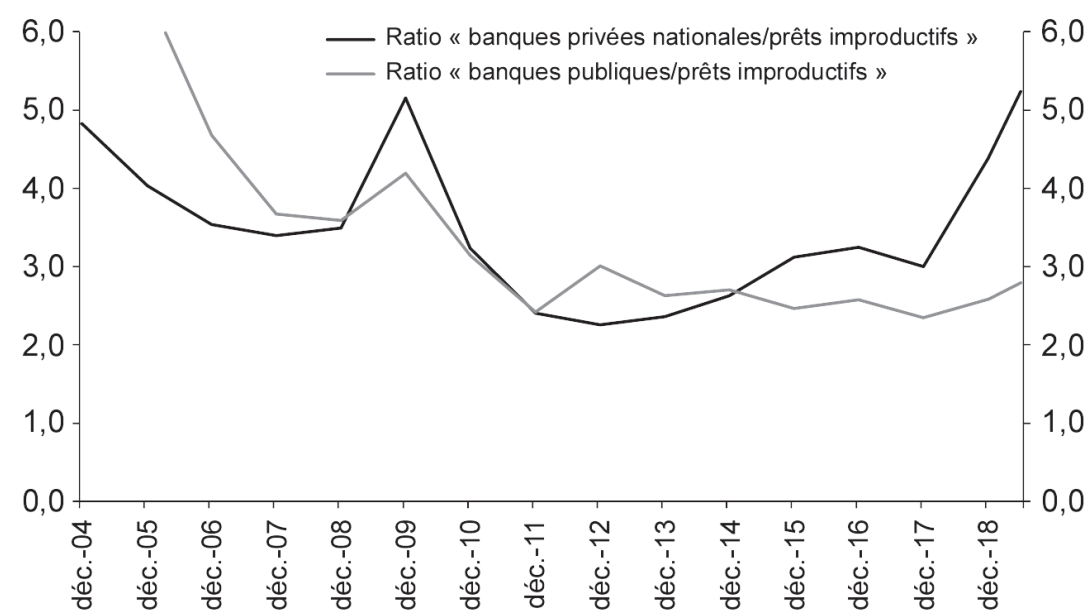

Note : sauf indication contraire, les agrégats s'entendent en livres turques.

Source des deux graphiques : données collectées auprès de l'Agence de réglementation et de surveillance bancaires. 


\section{Graphique 4 \\ Prêts syndiqués reçus par les banques, décembre 2002-juin 2019 [en milliards]}

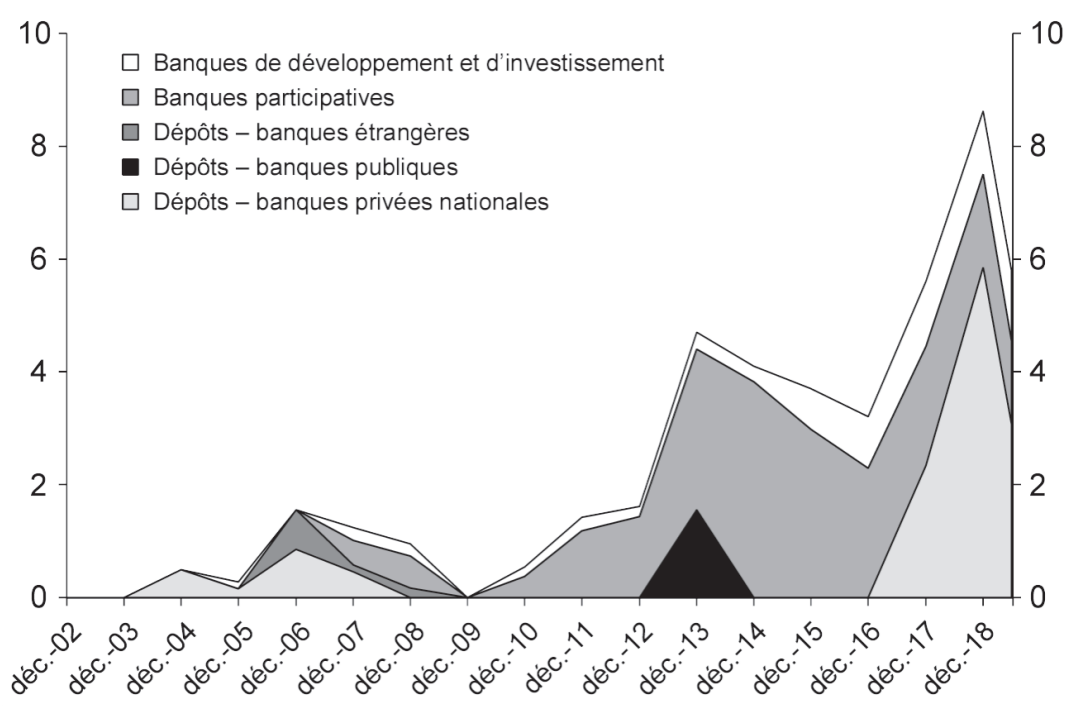

Notes : sauf indication contraire, les agrégats s'entendent en livres turques.

Dans l'ensemble, les agrégats du secteur ne sont pas le reflet de perspectives négatives ; autrement dit, en dépit de mauvaises perspectives macroéconomiques, le secteur bancaire lui-même n'est pas encore en crise. Nous maintenons une position critique dans la partie suivante afin de comprendre les limitations du secteur.

\section{LES BANQUES TURQUES AUJOURD'HUI : HISTOIRE D'UN SECTEUR DYNAMIQUE, MAIS TENDU}

Dans le contexte remarquable de l'environnement économique postLehman, les décideurs politiques et les universitaires turcs avaient presque coutume de dire que le secteur bancaire n'avait jamais été aussi robuste. D'un côté, ces déclarations peuvent être interprétées comme traduisant le soulagement ressenti à la suite des deux décennies problématiques dont sortait le secteur. D'un autre côté, elles ont été valables durant les deux premières années de la Grande Récession. Le secteur faisait preuve d'une grande résilience et n'imposait aucun risque qu'il aurait lui-même engendré.

Néanmoins certains choix politiques de l'autorité politique pourraient avoir introduit un élément de risque important à l'échelle du système. Un coup d'œil aux chiffres laisse entrevoir, si l'on compare aux $87 \%$ de 2010-2012, une hausse remarquable des actifs bancaires par 
rapport au PIB depuis 2013 jusqu'à atteindre un plateau de $105 \%$ (BDDK, 2019a). Le ratio " prêts/dépôts » du secteur (à l'exclusion des prêts consentis par les banques de développement et d'investissement) est d'environ $105 \%$ et cette valeur, bien qu'élevée par rapport aux $83 \%$ de 2010, reste inférieure à son pic de $115 \%$ en 2017 (BDDK, 2019a). Ces deux tendances, associées à l'expansion des prêts immobiliers, reflètent la récente euphorie qu'a connue le secteur du logement et de l'immobilier. Il convient de noter que lorsqu'ils sont traités distinctement pour les entités libellées en TRY et les entités libellées en devises, les ratios des prêts aux dépôts different considérablement. Ainsi, tandis que le ratio " prêts/dépôts " pour les entités libellées en TRY est passé de $89,1 \%$ en décembre 2018 à $144,7 \%$ en juin 2019, il est passé de 65,6\% à $83,7 \%$ pour les entités libellées en devises au cours de la même période.

\section{Euphorie dans le secteur du logement et de l'immobilier}

La loi n 6306 (adoptée le 16 mai 2012 et mise en œuvre le 31 mai 2012; Journal officiel, 2012), intitulée « Loi sur la transformation des zones exposées à un risque de catastrophe naturelle " ${ }^{6}$, semble avoir pour objectif premier de mobiliser des ressources destinées à renforcer le stock de bâtiments afin de limiter les pertes qui pourraient être provoquées par une catastrophe naturelle, en mettant l'accent sur l'éventualité d'un tremblement de terre dans la région de Marmara. À cette fin, la loi et les règlements connexes ont établi des moyens d'identifier, puis de renforcer ou reconstruire les structures susceptibles d'être endommagées lors d'un tremblement de terre. À première vue, cette introduction a été salutaire, dans la mesure où le processus de reconstruction était assorti de plusieurs mesures incitatives. Pourtant, la mise en œuvre de la loi a dépassé son cadre statutaire et s'est finalement transformée en euphorie nationale qui s'est d'abord manifestée dans les grandes zones métropolitaines. Qui plus est, lors de la reconstruction, les permis ont été révisés afin d'autoriser plus d'étages qu'auparavant. Une telle euphorie, mêlée aux dépenses d'infrastructure accrues du gouvernement central et des gouvernements locaux, a créé un environnement dans lequel l'autorité politique était en mesure d'intensifier l'activité économique et de gonfler le marché du crédit. Inutile de préciser que le secteur bancaire ne pouvait profiter de financements internationaux à des conditions plus favorables. De la même façon, des ratios " actifs bancaires/PIB " ou des ratios "prêts bancaires/dépôts " élevés sont la conséquence de choix politiques conscients qui pourraient n'avoir pas été bien calculés au départ. Le lecteur intéressé peut se référer au graphique 5 (infra) pour comprendre le poids de la construction dans les prêts.

Dans ce contexte, les bilans des banques sont devenus vulnérables au déclin soudain de l'activité de construction et/ou à une dépréciation suffisamment rapide de la livre turque par rapport à d'autres devises. Le premier aspect implique une diminution de l'activité économique et une 
hausse du taux de chômage, et donc une probabilité plus forte de prêts improductifs. En d'autres termes, le portefeuille de crédits du secteur est susceptible d'érosion, phénomène qui se produira vraisemblablement sur une longue période. Le second aspect a quant à lui une incidence immédiate sur les bilans. Il convient de noter qu'un ralentissement dans le secteur de la construction est observé depuis novembre 2017 et que la dépréciation de la livre turque a été plus rapide et plus volatile depuis août 2018.

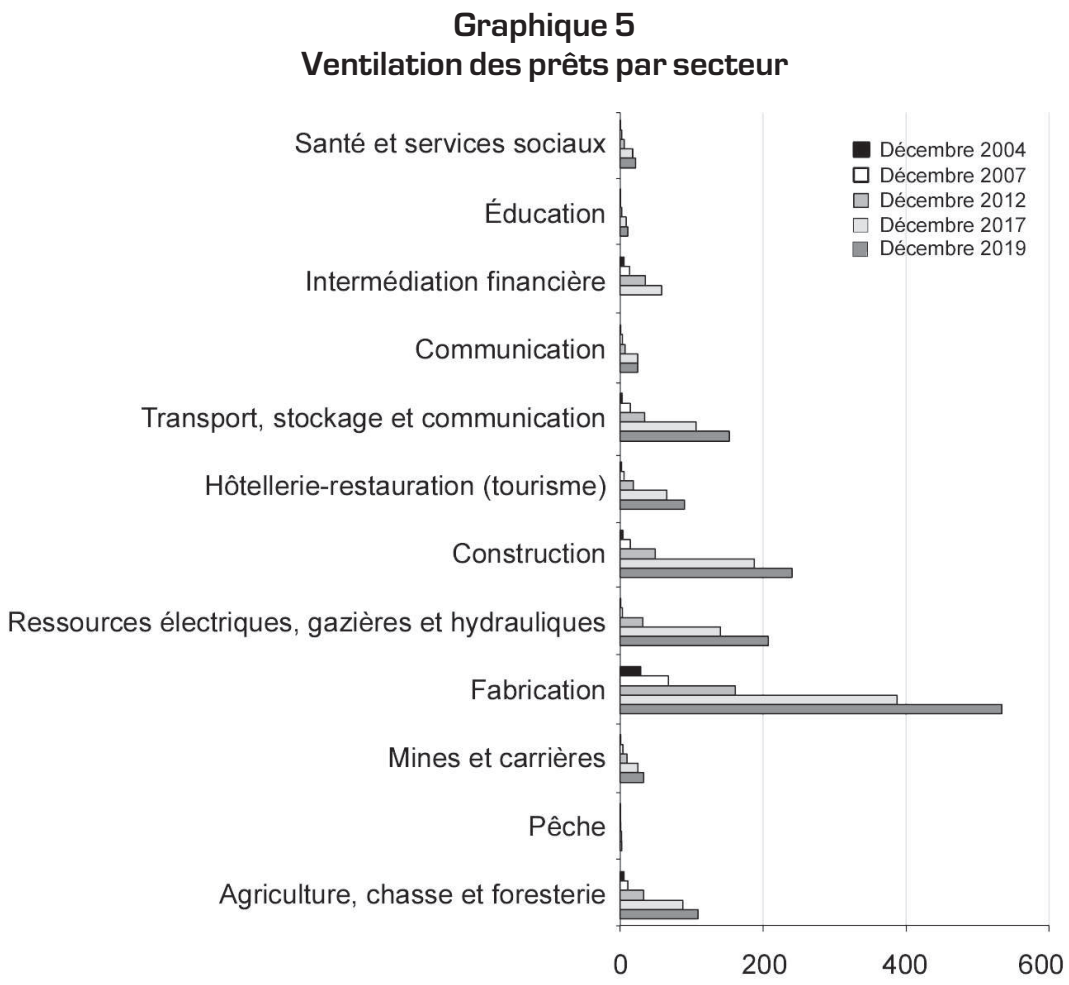

Notes : sauf indication contraire, les agrégats s'entendent en livres turques.

Source : données collectées auprès de l'Agence de réglementation et de surveillance bancaires.

\section{Formation d'un mauvais équilibre}

Manifestement, les vulnérabilités liées aux préférences de l'autorité politique semblent avoir joué un rôle prépondérant dans cette histoire. Ici, la mesure dans laquelle le secteur bancaire se conforme aux choix politiques du gouvernement ainsi que les raisons pour lesquelles il le fait sont deux points importants. La réponse au premier point présente peu d'intérêt : le fort niveau de conformité est tout simplement dû au fait que toutes les banques ont employé la même stratégie dans le jeu.

La réponse au second point, en revanche, demande à être précisée. En ce qui concerne le niveau de conformité fort, on pourrait évoquer 
une simple structure comparable à un jeu dans laquelle chaque banque choisit entre "financer la construction à peu de frais " ou "non ", compte tenu de ce qu'elle anticipe des actions du gouvernement et du comportement de ses concurrents. Dans un tel jeu, un équilibre superficiel se forme lorsque chaque banque fait la même chose, ici « financer la construction et l'immobilier ». Une explication plus simple, bien que réaliste, pourrait avoir trait aux tendances autocratiques croissantes du parti au pouvoir (Parti de la justice et du développement). Dans un environnement dépourvu de procédures de contrôle et de contrepoids suffisants, non seulement les banques se conforment aux choix du gouvernement, mais aussi elles les soutiennent aussi verbalement (les critiques, lorsqu'elles sont formulées, le sont de manière complaisante).

L'un dans l'autre, le climat turc post-2010 génère un équilibre fragile de dilemme collectif du côté des banques. De plus, cet échec collectif s'est développé de façon à introduire un cas classique de tragédie des biens communs qui se manifeste en termes de risque financier systémique, équivalent à une piètre diversification systémique du portefeuille d'activités. Indépendamment de la catégorie à laquelle une banque appartient - publique, privée locale ou étrangère -, le scénario se vérifie bien. Tandis que les banques publiques se conforment directement aux choix de l'autorité politique, les banques privées locales et les banques étrangères se comportent comme des suiveurs sur le marché.

\section{Un climat d'influence excessive du secteur public}

Pour en revenir à l'influence du secteur public, autrement dit de l'autorité politique, sur le secteur, un coup d'œil à l'allocation actuelle des ressources aux groupes bancaires montre le rôle important que joue l'État dans les activités bancaires. Ainsi, plus d'un tiers du secteur $(39,9 \%)$ est régi par l'État et trois banques détenues par l'État (Ziraat Bankas1, Halk Bankas1 et Vakiflar Bankas1) sont également des teneurs de marché désignés dans le domaine des titres de dette publique (les autres sont Akbank, Denizbank, HSBC, ING, QNB Finansbank, TEB, Garanti, Iş Bankas1 et Yapi ve Kredi Bankas1), représentant 39,8 \% des teneurs de marché. Le secteur public est donc censé avoir un niveau d'influence considérable sur l'activité bancaire et financière. En cela, et malgré que leurs données empiriques n'aient pas à notre connaissance été préalablement étudiées, les banques publiques ont une influence sur la transmission des taux directeurs au marché, reflétant leur fort ascendant dans le domaine de la formation des prix dans le secteur bancaire. Pourtant, réduire le problème aux parts de marché et aux mesures techniques ne refléterait que la moitié de la situation. L'autre moitié permet de discerner une série d'événements intéressants, comme en témoignent le cas de la Halk Bankas1 et l'enquête juridique à laquelle ont été soumises les banques après la tentative de coup d'État 
le 15 juillet 2016. Le cas de la Halk Bankas1 a été médiatisé dans le monde à la suite de l'incarcération de son directeur général adjoint aux États-Unis pour corruption d'un homme d'affaires résident turc d'origine iranienne, en lien avec la violation de l'embargo alors en vigueur contre l'Iran. Non ouvertement portée devant les tribunaux turcs, la violation de l'embargo contre l'Iran a souvent été considérée comme étant facilitée par un système de troc impliquant de l'or turc et du gaz naturel iranien. En cela, la Halk Bankas1 est supposée avoir pris part à la mise en place et à l'enregistrement des paiements s'y rapportant. Cela peut être considéré comme un cas manifeste d'abus perpétré par une autorité politique sur une banque publique.

L'enquête qui a visé les banques soupçonnées d'avoir été liées à la tentative de coup d'État de 2016 doit également faire l'objet d'un examen rigoureux afin de comprendre dans quelle mesure des instruments juridiques ont été utilisés contre des institutions financières, indépendamment du degré de rectitude observé par l'État. Lors de l'enquête, les administrations des banques ainsi que leurs clients professionnels et particuliers ayant été liés à la congrégation religieuse responsable de la tentative de prise du gouvernement ont été soumis à un contrôle juridique. À la fin du processus, plusieurs comptes bancaires ont été bloqués et les dirigeants de ces banques ont été remplacés. En résumé, l'indissociabilité des identités politiques et commerciales du côté des propriétaires de banques semble être une question cruciale.

\section{Propriété des grandes banques}

Outre l'indissociabilité des identités politiques et commerciales, une autre forme d'indissociabilité semble générer des sensibilités pour les banques, à savoir la détention presque naturelle des grandes banques privées par les principaux conglomérats commerciaux de Turquie. Il convient de noter que cette situation a été (perçue comme) un problème majeur avant 2000 en raison des risques qu'elle impliquait : l'allocation de crédits par une banque à ses sociétés sœurs au sein d'un conglomérat ou une version réciproque (interentreprises) de cela entre deux conglomérats détenant des banques n'étaient pas rares. Pourtant, cet écueil a été résolu par l'adoption de la loi bancaire ${ }^{0} 5411$. Un autre problème résultant de la détention de grandes banques par de grands conglomérats commerciaux est la sensibilité des banques détenues par des conglomérats aux préférences politiques parfois imprévisibles du gouvernement. On pourrait malheureusement résumer cette situation en disant que les banques sont " prises en otage par l'autorité politique ». Face à cette situation, le cas des banques ou des partenariats étrangers pourrait engendrer un peu de souplesse pour deux raisons : d'abord, les banques étrangères ont tendance à détenir une plus grande proportion de leurs actifs sous forme liquide ; 
ensuite, elles disposent de suffisamment de marge pour quitter la Turquie en cas d'intensification des problèmes politiques.

\section{Contestabilité dans le secteur}

Au vu du récent contexte historique présenté ci-dessus, un récapitulatif chiffré de la concurrence dans le secteur pourrait être nécessaire. À cette fin, nous examinons brièvement certains indicateurs habituels de la concentration de marché en ce qui concerne, d'une part, les actifs et, d'autre part, les dépôts. L'indice Herfindahl-Hirschman (IHH), l'IHH normalisé $\left(\mathrm{IHH}^{*}\right)$, le ratio de concentration basé sur quatre entreprises (CR4) et le ratio de concentration basé sur huit entreprises (CR8) sont calculés pour le secteur, tel que présenté dans les parties supérieures des tableaux 2 et 3 (infra). Nous répétons ensuite cet exercice après élimination des huit premières banques afin de déterminer la concentration

\section{Tableau 1}

Quelques parts de marché sélectionnées, mars 2019

[en $\%$ des actifs totaux]

\begin{tabular}{ll}
\hline État dans le secteur & $39,9 \%$ \\
État dans le groupe des teneurs de marché & $39,8 \%$ \\
Banques étrangères dans le secteur & $24,2 \%$ \\
Banques étrangères dans le groupe des teneurs de marché & $23,3 \%$ \\
Banques privées nationales dans le secteur & $35,9 \%$ \\
Banques privées nationales dans le groupe des teneurs de marché & $36,9 \%$ \\
\hline
\end{tabular}

Sources : calculs de l'auteur (les données brutes ont été collectées auprès de l'Association des banques de Turquie].

Tableau 2

Concentration des actifs bancaires

\begin{tabular}{|c|c|c|c|c|c|}
\hline & 2002 & 2007 & 2012 & 2017 & 2019 \\
\hline & \multicolumn{5}{|c|}{ (a) Toutes les banques } \\
\hline Indice Herfindahl-Hirschman (IHH) & 0,104 & 0,098 & 0,092 & 0,088 & 0,087 \\
\hline Indice Herfindahl-Hirschman normalisé ( $\left.\mathrm{IHH}^{*}\right)$ & 0,082 & 0,076 & 0,070 & 0,068 & 0,067 \\
\hline Ratio de concentration fondé sur quatre entreprises (CR4) & 0,545 & 0,540 & 0,505 & 0,468 & 0,460 \\
\hline \multirow[t]{2}{*}{ Ratio de concentration fondé sur huit entreprises (CR8) } & 0,823 & 0,820 & 0,805 & 0,794 & 0,783 \\
\hline & \multicolumn{5}{|c|}{ (b) En excluant les huit premières } \\
\hline Indice Herfindahl-Hirschman (IHH) & 0,077 & 0,089 & 0,096 & 0,094 & 0,095 \\
\hline Indice Herfindahl-Hirschman normalisé ( $\left.\mathrm{IHH}^{*}\right)$ & 0,048 & 0,061 & 0,069 & 0,069 & 0,072 \\
\hline Ratio de concentration fondé sur quatre entreprises (CR4) & 0,437 & 0,534 & 0,546 & 0,545 & 0,546 \\
\hline Ratio de concentration fondé sur huit entreprises (CR8) & 0,717 & 0,745 & 0,755 & 0,732 & 0,708 \\
\hline
\end{tabular}

Notes : dans la partie (a), quatre mesures de concentration sont fournies pour les actifs bancaires. La partie (b) répète l'exercice pour un sous-ensemble de banques qui exclut les données des huit premières banques. Les chiffres de 2019 reflètent la situation à la fin de mars 2019.

Sources : calculs de l'auteur (les données brutes ont été collectées auprès de l'Association des banques de Turquie). 
Tableau 3

Concentration des dépôts bancaires

\begin{tabular}{lrrrrr}
\hline & 2006 & 2007 & 2012 & 2017 & 2019 \\
\cline { 2 - 6 } & \multicolumn{5}{c}{ (a) Toutes les banques } \\
\hline Indice Herfindahl-Hirschman (IHH) & 0,089 & 0,088 & 0,087 & 0,086 & 0,088 \\
Indice Herfindahl-Hirschman normalisé (IHH*) & 0,064 & 0,064 & 0,065 & 0,066 & 0,069 \\
Ratio de concentration fondé sur quatre entreprises (CR4) & 0,506 & 0,498 & 0,472 & 0,460 & 0,472 \\
Ratio de concentration fondé sur huit entreprises (CR8) & 0,780 & 0,780 & 0,787 & 0,775 & 0,787 \\
\hline & (b) En excluant les huit premières \\
\hline Indice Herfindahl-Hirschman (IHH) & 0,103 & 0,104 & 0,102 & 0,096 & 0,102 \\
Indice Herfindahl-Hirschman normalisé (IHH*) & 0,071 & 0,074 & 0,074 & 0,069 & 0,077 \\
Ratio de concentration fondé sur quatre entreprises (CR4) & 0,569 & 0,579 & 0,559 & 0,553 & 0,561 \\
Ratio de concentration fondé sur huit entreprises (CR8) & 0,806 & 0,805 & 0,799 & 0,741 & 0,744 \\
\hline
\end{tabular}

Notes : dans la Partie (a), quatre mesures de concentration sont fournies pour les dépôts bancaires. La Partie (b) répète l'exercice pour un sous-ensemble de banques qui exclut les données des huit premières banques. Les chiffres de 2019 reflètent la situation à la fin de mars 2019.

Sources : calculs de l'auteur (les données brutes ont été collectées auprès de l'Association des banques de Turquie).

des banques relativement petites. D'après nos calculs, la concentration de marché semble avoir diminué seulement légèrement au fil des ans, à la fois pour le secteur dans son ensemble et pour le secteur à l'exclusion des huit premières banques. En d'autres termes, la composition du marché telle que mesurée par le nombre d'entités et l'allocation des ressources demeure presque inchangée dans le temps. Les opérations des banques étrangères en Turquie exigent des partenariats avec les entreprises bancaires locales dans la mesure du possible.

\section{CONCLUSION}

Jusqu'ici, notre récit fait la lumière sur la décennie d'euphorie de croissance économique portée par le secteur de la construction et sur la tendance de croissance du crédit qui en a résulté, sur le dilemme collectif des banques et sur la contraction de leur capacité de manœuvre. Parallèlement à cela, les perspectives des entités bancaires étrangères d'opérer de manière sécurisée en Turquie s'avèrent limitées. En effet, le 19 juillet 2019, alors que la rédaction de cet article était en cours, l'agence internationale de notation de crédit Fitch Ratings a baissé les notes de douze banques turques sous contrôle étranger et de deux banques publiques de $\mathrm{BB}-\mathrm{à} \mathrm{B}+$, en insistant sur leurs perspectives "négatives". Pour les premières, l'agence a justifié sa décision en évoquant la probabilité accrue d'une intervention du gouvernement face à la détérioration des finances extérieures de la Turquie. Pour les deux banques publiques, la raison avancée était la capacité limitée des 
décideurs politiques à faire face aux risques de change ${ }^{7}$. Associée à l'abaissement de la note du pays par Fitch un peu plus tôt le 12 juillet 2019, la baisse des notes des banques a tiré la sonnette d'alarme.

À la même époque, Yesilada (2019) a souligné la possibilité d'une " nouvelle approche d'Istanbul ", en faisant référence à la restructuration massive des prêts accordés au secteur privé à la suite de la crise financière de février 2001. En quelques mots, l'Approche d'Istanbul était un programme de sauvetage conçu pour le secteur des sociétés non financières ainsi que pour le secteur bancaire de Turquie. Partageant une perspective de résolution similaire à celle de la Suède après sa crise de 1993, au cours de laquelle les bilans du secteur des sociétés non financières et du secteur bancaire ont été considérés conjointement, l'Approche d'Istanbul a aidé la Turquie à compenser la détérioration de l'économie ${ }^{8}$. Les remarques relatives à une nouvelle Approche d'Istanbul peuvent ainsi être interprétées comme un signe de détérioration des perceptions de l'état de santé des banques.

L'un dans l'autre, la normalisation du secteur bancaire nécessiterait une fois encore d'attendre une nouvelle décennie. En effet, ce processus est étroitement lié à la normalisation de la configuration politique multipartite et du rétablissement de l'autonomie et des compétences du côté des bureaucrates chargés de la prise de décisions.

Loin des traditions universitaires, il serait intéressant de clore cette étude par quelques cas isolés ayant récemment fait surface. Ainsi, comme Bloomberg News l'a porté à l'attention du public au moment où cet article faisait l'objet d'ultimes révisions avant publication, onze anciens dirigeants de banques non publiques ont annoncé avoir été renvoyés ces deux dernières années sur ordre des régulateurs bancaires, lesquels œuvrent sous la houlette du ministère du Trésor et des Finances. En dépit de son caractère pour l'heure anecdotique, cette situation peut être considérée comme un indice sérieux de l'existence de problèmes sous-jacents. De nouvelles analyses, dès que davantage de données seront disponibles, permettraient de mettre au jour les problèmes du secteur ainsi que des solutions justes.

\section{NOTES}

1. Suivant la tradition classique, un système financier (en particulier bancaire) qui fonctionne correctement facilite les fonctions (1) d'économies d'échelle en matière de transactions et d'information, (2) d'assurance et (3) de transformation des échéances.

2. Dans les années 1990, la Turquie était dépourvue d'autorités de surveillance compétentes, de cadre réglementaire et d'infrastructure juridique et institutionnelle ; de plus, les règlements prudentiels existants étaient mal appliqués. Les coûts énormes occasionnés par la crise de février 2001 ont mis en lumière l'importance de ces lacunes (Togan et al., 2010). 
3. Le Comité de Bâle a été établi à la fin de 1974 par les gouverneurs des banques centrales du " groupe des Dix ». En 1988, le Comité a introduit un système de mesure des capitaux souvent appelé " Accord de Bâle sur les fonds propres (Bâle I) ». À la suite des critiques formulées à l'encontre de Bâle I, le Comité de Bâle a révisé l'Accord sur les capitaux, annoncé Bâle II en juin 2004, et a prévu sa mise en œuvre avant la fin de 2006 dans les pays du G10. Bâle II comporte les grands piliers de (1) l'exigence de fonds propres, de (2) la procédure de surveillance de la gestion des fonds propres et de (3) la discipline de marché.

4. Sauf indication contraire, les parties 3 et 4 s'appuient sur des données allant jusqu'au second trimestre 2019 inclus (fin juin 2019) et dont les sources sont indiquées dans les visuels présentés. Le lecteur intéressé trouvera un ensemble de chiffres clés relatifs aux banques de Turquie en annexe.

5. Le ratio "TRY/devises » tel que nous l'avons mis en œuvre dans cet article mesure la réciproque de la dollarisation ; autrement dit, une diminution du ratio "TRY/devises " indique des préférences marquées pour une substitution monétaire.

6. Traduction en français d'après une traduction en anglais de l'auteur.

7. Les banques touchées par une baisse de leur note étaient : Garanti, Yapi ve Kredi, TEB, QNB Finansbank, ING Bank, la banque participative Kuveyt Turk, Alternatifbank, la banque participative Turkiye Finans, Burgan Bank, ICBC Turkey Bank, BankPozitif Kredi ve Kalkinma Bankası, Denizbank, Turk Eximbank et Turkiye Kalkinma Bankası.

8. Une restructuration similaire a été étudiée de manière approfondie au début de 2009 par la Fondation turque de recherche sur la politique économique (TEPAV) en collaboration avec le ministre chargé de la coordination économique, Nazim Ekren, l'Association des banques de Turquie et l'Union turque des chambres et bourses de commerce. Toutefois, le climat actuel a fait que les projections et les propositions politiques ont été laissées à l'abandon. Le cadre de ces efforts est fourni dans TEPAV (2009).

\section{BIBLIOGRAPHIE}

AvCI B. et YüCEL E. (2017), « Effectiveness of Monetary Policy: Evidence from Turkey », Eurasian Economic Review, $\mathrm{n}^{\circ}$ 7, pp. 179-213, DOI : https://link.springer.com/article/10.1007/s40822-017-0068-y.

BDDK (Agence turque de réglementation et de surveillance bancaires) (2019a), Principaux indicateurs du secteur bancaire turc, mars.

Bloomberg News (2019), "Top Turkey Bankers Say They Were Fired on Regulators' Orders ", 15 décembre 2019, 8:03 AM EST, mis à jour le 15 décembre 2019, 12:18 PM EST, https://www.bloom berg.com/amp/news/articles/2019-12-15/top-turkish-bankers-say-they-were-fired-on-orders-ofregulators?_twitter_impression=true. Dernière consultation : 19 décembre 2019.

Guler B. (2019), «Copenhagen Revisited: Operational Measures for Functioning Market Economy Criterion ", manuscrit non publié.

Journal OfFICIEL (2001), Loi sur la banque centrale no 4651, numéro 24393, 5 mai.

JoURNAL OFFICIEL (2005), Loi bancaire n $\mathrm{n}^{\circ} 5411$, numéro 25983 , $1^{\text {er }}$ novembre.

JOURNAL OFFICIEL (2012), Loi sur la transformation des zones exposées à un risque de catastrophe naturelle n 6306, numéro 28309, 31 mai.

TBB (Association des banques de Turquie), Système d'interrogation de données, https://www.tbb.org.tr /en/banks-and-banking-sector-information/data-query-system/21.

TEPAV (2009), "Crisis Measures for the Turkish Economy: a Framework Proposal ", consulté pour la dernière fois à l'adresse https://www.tepav.org.tr/en/yayin/s/34 le 15 septembre 2019, signé par le Groupe de travail TEPAV sur la crise mondiale (E. Yucel, E. Caglar, F. Ozatay, G. Sak, H. Pamuk, H. H. Yilmaz, H. Ersel, O. Acar, S. Kalkan, S. Sayek, S. Serdengecti et Z. Tatar).

Togan S., Berument H. et Ersel H. (2010), "Liberalization of Banking Services ", in Togan S., Economic Liberalization and Turkey, chapitre 7, Routledge Political Economy of the Middle East and North Africa, New York, pp. 153-187.

YesILADA A. (2019), «A new Istanbul Approach Is a Approaching », 17 juillet. 


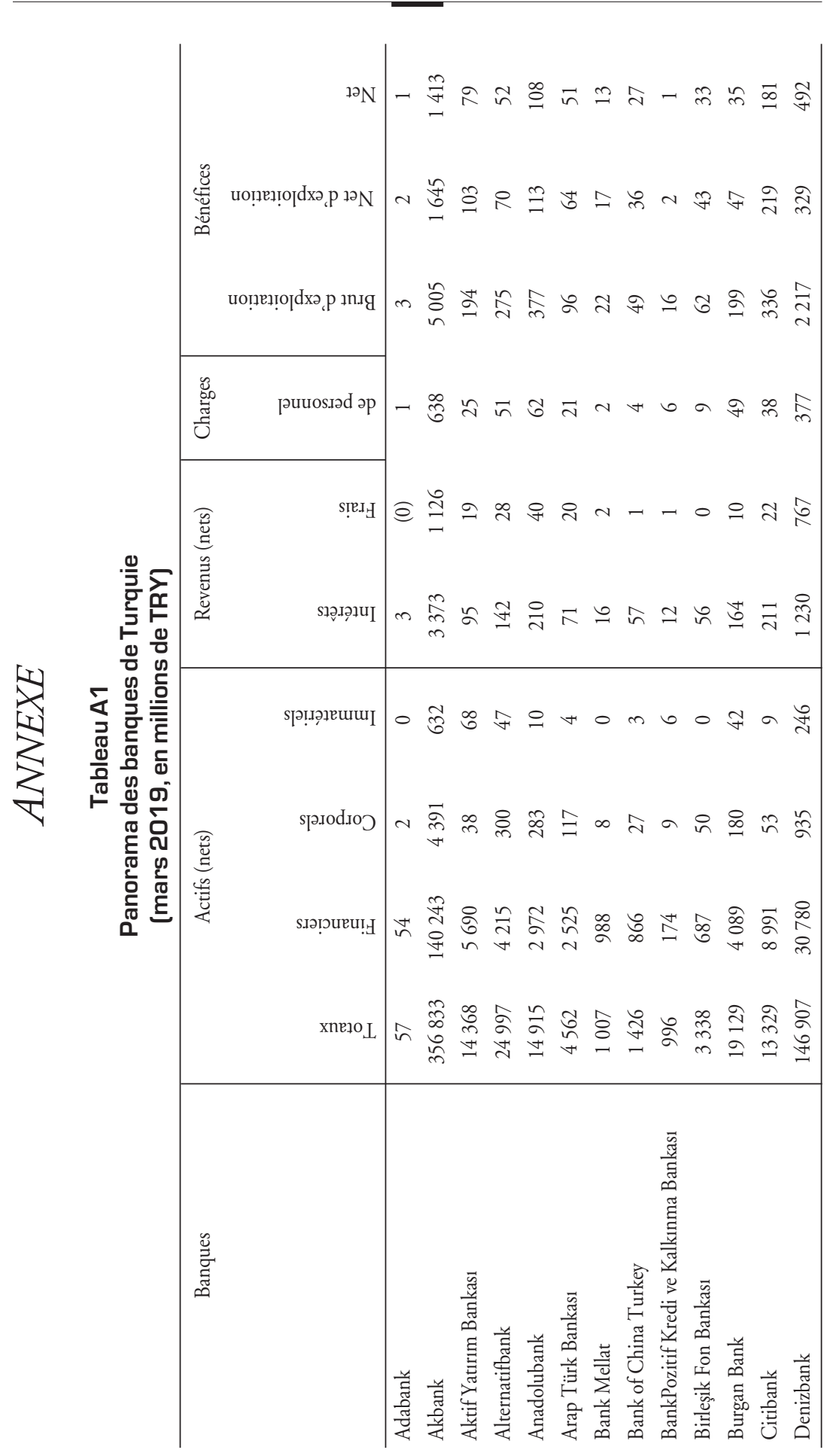




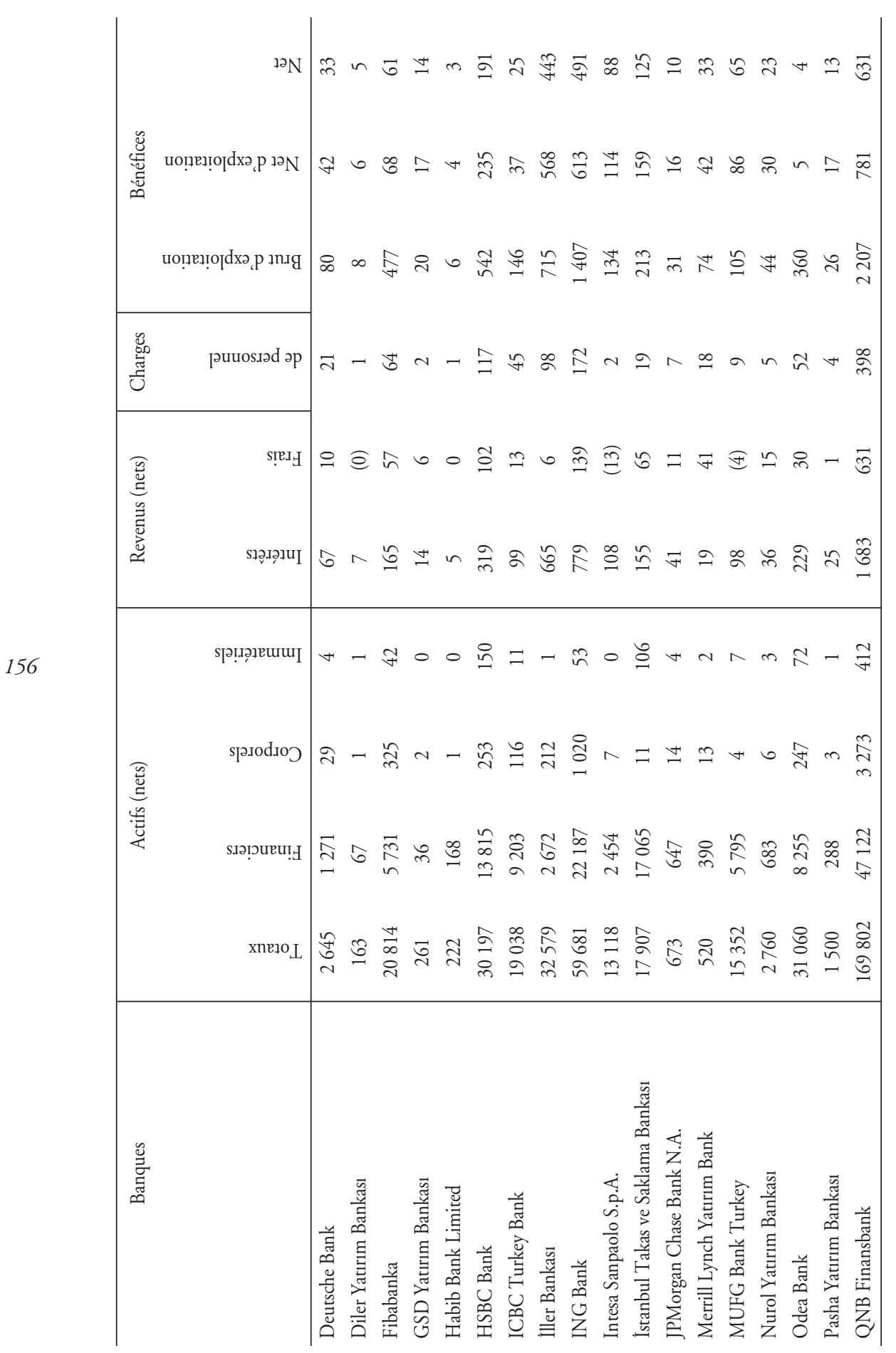




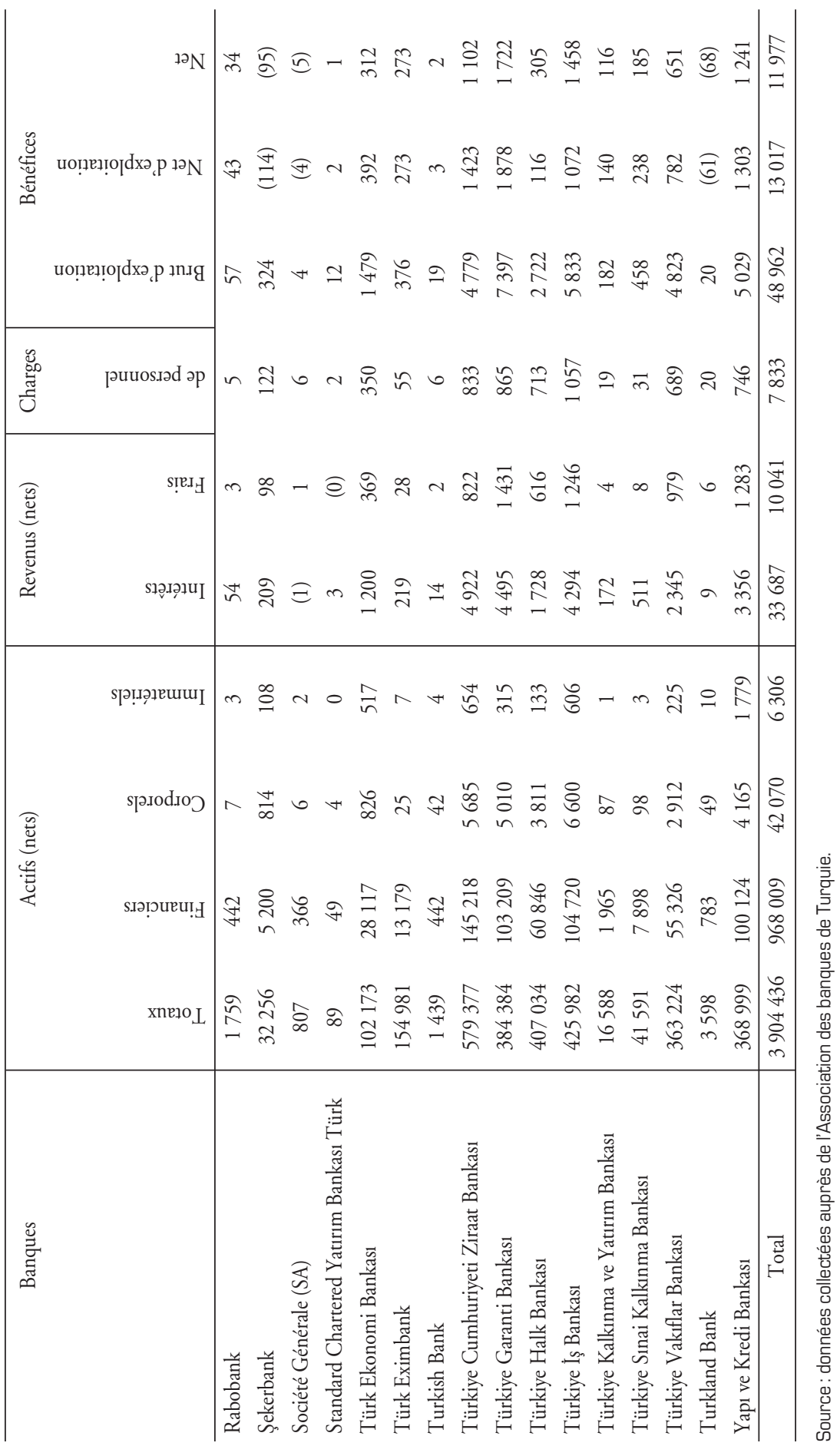

